\title{
Aspergillus mastoiditis, presenting with unexplained progressive otalgia, in an immunocompetent (older) patient
}

\author{
Aukje van Tol $\cdot$ Jeroen van Rijswijk
}

Received: 29 June 2008 / Accepted: 11 November 2008 / Published online: 4 December 2008

(C) The Author(s) 2008. This article is published with open access at Springerlink.com

\begin{abstract}
Aspergillus mastoidits and skull-base osteomyelitis are extremely rare, even in immunocompromised patients. We report a case of an 81-year-old immunocompetent man, who underwent a mastoidectomy because of unexplained, progressive otalgia in spite of a noninflamed and air-containing middle-ear space. Histopathology yielded Aspergillus fumigatus. When confronted with otitis with an unexpected clinical course a high index of suspicion is required to facilitate early diagnosis and appropriate therapy of a potential lethal Aspergillus infection, even in immunocompetent patients. This seems to be more so in older patients with an open middle-ear cavity and/or when there is facial nerve involvement.
\end{abstract}

Keywords Aspergillus · Matoiditis - Immunocompetent . Otitis

\section{Introduction}

Invasive skull-base mastoiditis, also known as malignant or necrotizing otitis externa, typically affects immunocompromised patients (diabetes, leukemia, AIDS, prior treatment

\section{A. van Tol}

Department of Otorhinolaryngology,

Head and Neck Surgery, Erasmus Medical

Center Rotterdam, Rotterdam, The Netherlands

J. van Rijswijk

Department of Otorhinolaryngology,

Head and Neck Surgery and Facial Plastics,

Van Weel-Bethesda Hospital, Dirksland, The Netherlands

J. van Rijswijk ( $\square)$

Freudenbergstrasse 3, 8044 Zurich, Switzerland

e-mail: jeroen@vanryswyk.nl with cytotoxic medication and or corticosteroids) and is usually caused by Pseudomonas aeruginosa.

Although being an uncommon condition, it is often well recognized and at one moment differentiated from an 'ordinary' otitis due to alarming (progressive) symptoms such as extreme/deep otalgia, vertigo-hearing loss (labyrinthitis), facial nerve dysfunction and sometimes dysfunction of other nerves of the cerebellopontine angle (Gradenigo syndrome).

Although human exposure to Aspergillus is common, infections are infrequent. Aspergillus mastoiditis and skullbase osteomyelitis are extremely rare, even in immunocompromised patients [5], the first patient being described in 1985 [8]. We report a case of invasive Aspergillus fumigatus skull-base mastoiditis in an immunocompetent patient, only first recognized at surgery.

\section{Case report}

An 81-year-old man presented with a 5-week history of right-sided otalgia and otorrhoea (Table 1). His hearing in the right ear was subjectively reduced; there was no history of tinnitus or vertigo. Examination revealed whitish debris filling the right external auditory canal. The left ear was normal. His medical history included a symmetrical presbyacusis, for which he had been wearing hearing aids bilaterally since 1999 . He did not take any medication. Initially, the diagnosis of a right-sided external otitis was made and the patient was treated with oral antibiotics and acetic acid ear drops.

One month later the patient returned with progressive, intermittent severe/undurable, right-sided otalgia. Examination showed some mild otorrhoea as well as a quiet, central perforation in the tympanic membrane 
Table 1

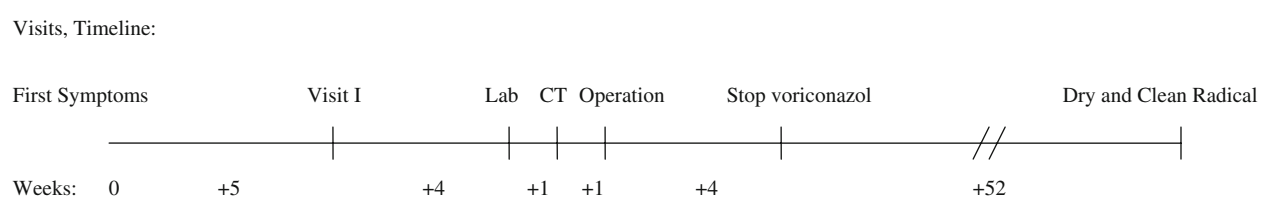

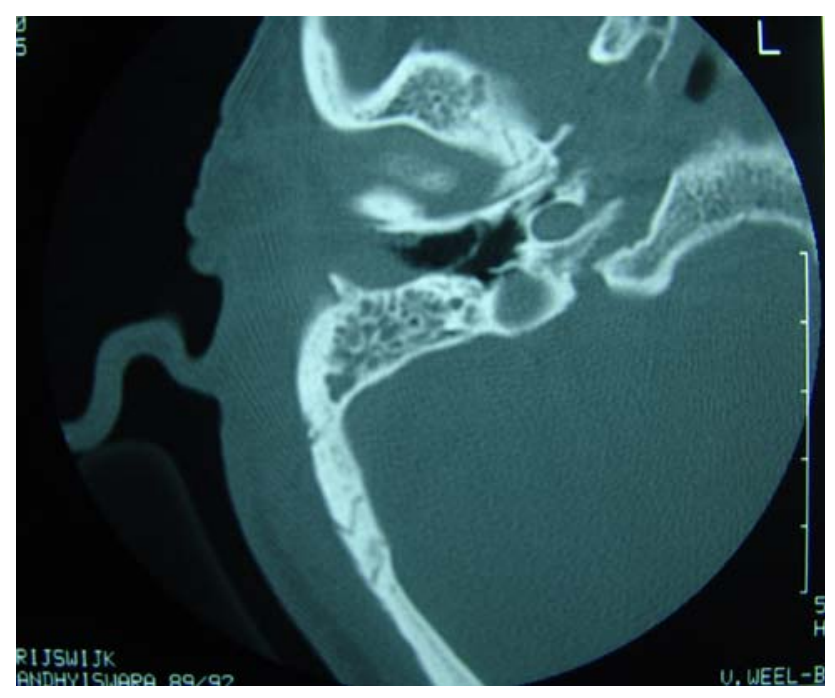

Fig. 1 Axial CT scan of the right temporal bone showing complete opacification of the mastoid air cells, an air-containing middle ear and swelling of the soft tissues surrounding the ear

with a noninflamed and air-containing middle ear space. The ear canal was slightly edematous. No granulation tissue could be seen in the external auditory canal or middle ear by microscopic examination. There was no post-auricular fluctuation and no erythema, tenderness or edema over the mastoid area. Facial function was normal. The Valsalva maneuver was negative.

Initial laboratory workup showed signs of an infection [ESR $46 \mathrm{~mm} / \mathrm{h}$, CRP $21.3 \mathrm{mg} / \mathrm{l}$, normal WBC $\left(8.6 \times 10^{9} /\right.$; $16 \%$ lymphocytes)]. Liver enzymes were normal. Fasting blood sugar was $6.1 \mathrm{mmol} / \mathrm{l}$. Anti-dsDNA, ENA, anti-proteinase 3 and anti-myeloperoxidase were all negative. Tone and speech audiometry showed a superimposed conductive hearing loss of the right ear. Because of the ununderstood invalidating earache (not matching with the quite external auditory canal, tympanic membrane and middle ear) a computed tomography (CT) scan was planned. This scan (1 week later) demonstrated complete opacification of the right mastoid air cells, swelling of the soft tissues surrounding the ear but no signs of bony erosion (Fig. 1).

Considering the aforementioned and the still progressive, sometimes undurable otalgia, despite the use of broad spectrum antibiotics and strong analgesics, we decided to perform a cortical mastoidectomy to obtain mastoid-material for histopathologic investigation.
At surgery subcutaneous edema and a black discoloration of the mastoid cortex and the bony ear canal was encountered. This was the first time invasive fungal mastoiditis or other bone infiltrating pathology was suspected. Therefore, we decided to perform directly a canal wall down (radical mastoidectomy) procedure to remove as much infected bone as possible and to ensure a good drainage. Purulent fluid and widespread granulation tissue filled the mastoid air cells. Intraoperative specimens of tissue were sent for culture and histopathological analysis.

After surgery an infectious disease consult was obtained to medically complement surgical eradication of the fungal infection. Postoperatively, the patient was treated with local miconazol dressing on cotton strips and oral voriconazol (200 mg twice daily) for 4 weeks.

Histolopathological analysis revealed necrosis, inflammation/granulocytes, and numerous septate fungal hyphae. Cultures of mastoid material obtained during surgery yielded Aspergillus fumigatus.

At follow-up visits after surgery, a well-healed incision was seen and the mastoid cavity re-epithelialized normally in several months with no evidence of residual or persistent disease by microscopic examination (last visit 1 year after surgery).

\section{Discussion}

Mycotic infections of the head and neck region are uncommon. Infections of the external auditory canal and paranasal sinuses are the most encountered sites.

The classification of Aspergillus infections has been described for paranasal sinus aspergillosis, and has been expanded to include temporal bone infection [5].

Documented cases of Aspergillus mastoiditis are exceedingly rare and all but six $[1-4,6,7]$ of the reported cases to date involved immunocompromised patients.

Patients with invasive aspergillosis can have similar clinical presentation and physical findings as an infection caused by Pseudomonas aeruginosa. Failure to identify Aspergillus as the causative pathogen of invasive temporal bone infection is the principal reason for delay in initiating potentially life-saving therapy.

In our patient mainly the mastoid was involved with the infection and not so the well-aerated middle ear (Fig. 1). This 
unusual finding might be explained with an obstruction of the aditus ad antrum through pathologic tissue (granulation tissue) in combination with the tympanic membrane perforation [10].

Almost all patients with documented invasive external otitis resulting from Aspergillus reported in literature were noted to have facial nerve involvement. Evidence of facial nerve involvement in patients with mastoiditis-particularly those who are immunocompromised and do not respond to antibiotics-should cause one to consider Aspergillus in the differential diagnosis [1-6, 8].

Another noteworthy co-incidence in the seven documented immunocompetent patients with Aspergillus mastoiditis to date (our patient included) is their old age (52-85, average 75 years) and the fact that they all had a port d'entrée to the tympanic cavity or mastoid: six had a tympanic membrane perforation $[1-4,6]$ and the seventh (with the age of 52) was already known with a cholesteatoma (for which she refused surgery) 9 years before the mastoiditis [7].

Treatment of Aspergillus mastoiditis is threefold. Aggressive surgical debridement and resection is required and antifungal therapy should be instituted once the diagnosis is made. Hyperbaric oxygen therapy should be considered post-operative although clear evidence to demonstrate effectiveness is still missing [9].

Further, attempt should be made to find and control any underlying immunologic conditions.

\section{Conclusion}

Mastoiditis caused by Aspergillus species is very uncommon. Because of the invasive nature of this life threatening disease, prompt diagnosis and aggressive management including surgical debridement and antifungal therapy are necessary.

When confronted with otitis/ear complaints with an unexpected clinical course a high index of suspicion is required to facilitate early diagnosis and appropriate therapy of a potential lethal Aspergillus infection, even in immunocompetent patients. This seems the more so in older patients with an open middle ear cavity (tympanic membrane perforation, cholesteatoma) and/or when there is facial nerve involvement.

This is for we know the seventh immunocompetent patient with invasive Aspergillus mastoiditis described in the world literature.

Conflict of interest statement Both authors indicate that they do not have a financial relationship with the organization that sponsored the research. Also there has not been any organization that sponsored the research.

Open Access This article is distributed under the terms of the Creative Commons Attribution Noncommercial License which permits any noncommercial use, distribution, and reproduction in any medium, provided the original author(s) and source are credited.

\section{References}

1. Bickley LS, Betts RF, Parkins CW (1988) Atypical invasive external otitis from Aspergillus. Arch Otolaryngol Head Neck Surg 114:1024-1028

2. Bryce GE, Phillips P, Lepawsky M, Gribble MJ (1997) Invasive Aspergillus tympanomastoiditis in an immunocompetent patient. J Otolaryngol 26:266-269

3. Cunningham M, Yu VL, Turner J, Curtin H (1988) Necrotizing otitis externa due to Aspergillus in an immunocompetent patient. Arch Otolaryngol Head Neck Surg 114:554-556

4. Gordon G, Giddings NA (1994) Invasive otitis externa due to Aspergillus species: case report and review. Clin Infect Dis 19:866-870

5. Hall PJ, Farrior JB (1993) Aspergillus mastoiditis. Otolaryngol Head Neck Surg 108:167-170

6. Menachof MR, Jackler RK (1990) Otogenic skull base osteomyelitis caused by invasive fungal infection. Otolaryngol Head Neck Surg 102:285-289

7. Ohki M, Ito K, Ishimoto S (2001) Fungal mastoiditis in an immunocompetent adult. Eur Arch Otorhinolaryngol 258:106-108

8. Petrak RM, Pottage JC, Levin S (1985) Invasive external otitis caused by Aspergillus fumigatus in an immunocompromised patient. J Infect Dis 151:196

9. Phillips JS, Jones SEM (2005) Hyperbaric oxygen as an adjuvant treatment for malignant otitis externa. Cochrane Database of Systematic Reviews:Art. No.: CD004617. doi: 004610.001002/ 14651858.CD14004617.pub14651852

10. Schachern P, Paparella MM, Sano S, Lamey S, Guo YH (1991) A histopathological study of the relationship between otitis media and mastoiditis. Laryngoscope 101:1050-1055 